\title{
On Producing High and Early Result Throughput in Multi-join Query Plans
}

\author{
Justin J. Levandoski, Member, IEEE, Mohamed E. Khalefa, Member, IEEE, \\ and Mohamed F. Mokbel, Member, IEEE
}

\begin{abstract}
This paper introduces an efficient framework for producing high and early result throughput in multi-join query plans. While most previous research focuses on optimizing for cases involving a single join operator, this work takes a radical step by addressing query plans with multiple join operators. The proposed framework consists of two main methods, a flush algorithm and operator state manager. The framework assumes a symmetric hash join, a common method for producing early results, when processing incoming data. In this way, our methods can be applied to a group of previous join operators (optimized for single-join queries) when taking part in multi-join query plans. Specifically, our framework can be applied by (1) employing a new flushing policy to write in-memory data to disk, once memory allotment is exhausted, in a way that helps increase the probability of producing early result throughput in multi-join queries, and (2) employing a state manager that adaptively switches operators in the plan between joining in-memory data and disk-resident data in order to positively affect the early result throughput. Extensive experimental results show that the proposed methods outperform the state-of-the-art join operators optimized for both single and multi-join query plans.
\end{abstract}

Index Terms-Database Management, Systems, Query processing.

\section{INTRODUCTION}

$\mathrm{T}$ RADITIONAL join algorithms (e.g., see [1], [2], [3]) are designed with the implicit assumption that all input data is available beforehand. Furthermore, traditional join algorithms are optimized to produce the entire query result. Unfortunately, such algorithms are not suitable for emerging applications and environments that require results as soon as possible. Such environments call for a new non-blocking join algorithm design that is: (1) applicable in cases where input data is retrieved from remote sources through slow and bursty network connections, (2) optimized to produce as many early results as possible (i.e., produce a high early result throughput) in a nonblocking fashion, while not sacrificing performance in processing the complete query result, and (3) operates in concert with other non-blocking operators to provide early (i.e., online) results to users or applications. In other words, any blocking operation (e.g., sorting) must be avoided when generating of online results.

Examples of applications requiring online results include web-based environments, where data is gathered from multiple remote sources and may exhibit slow and bursty behavior [4]. Here, a join algorithm should be capable of producing results without waiting for the arrival of all input data. In addition, web users prefer early query feedback, rather than

- J.J. Levandoski, M.E. Khalefa, and M.F. Mokbel are with the Department of Computer Science and Engineering, Unversity of Minnesota - Twin Cities, 200 Union Street SE, Minneapolis, MN 55455. E-mail: \{justin,khalefa,mokbel\}@cs.umn.edu waiting an extended time period for the complete result. Another vital example is scientific experimental simulation, where experiments may take up to days to produce large-scale results. In such a setting, a join query should be able to function while the experiment is running, and not have to wait for the experiment to finish. Also, scientists prefer to receive early feedback from long-running experiments in order to tell if the experiment must halt and be restarted with different settings due to unexpected results [5], [6]. Other applications that necessitate the production of early results include streaming applications [7], [8], workflow management [9], data integration [10], parallel databases [11], spatial databases [12], sensor networks [13], and moving object environments [14].

Toward the goal of producing a high early result throughput in new and emerging online environments as those just described, several research efforts have been dedicated to the development of nonblocking join operators (e.g., see [15], [16], [17], [12], [18], [19], [20], [8], [21]). However, with the exception of [17], these algorithms focus on query plans containing a single join operator. The optimization techniques employed by these join operators focus on producing a high early result throughput locally, implying that each operator is unaware of other join operators that may exist above or below in the query pipeline. Optimizing for local throughput does not necessarily contribute to the goal of producing a high overall early result throughput in multi-join query plans.

In general, the ability of join queries to produce high early result throughput in emerging environments lies in the optimization of two main tasks: 
(1) The ability to handle large input sizes by flushing the least beneficial data from memory to disk when memory becomes exhausted during runtime, thus allowing new input tuples to produce early results, and (2) The ability to adapt to input behavior by producing results in a state (e.g., in-memory or on-disk) that positively affects early result throughput. In this paper, we explore a holistic approach to optimizing these two tasks for multi-join query plans by proposing two methods that consider join operators in concert, rather than as separate entities. The first method is a novel flushing scheme, named AdaptiveGlobalFlush, that intelligently selects a portion of in-memory data that is expected to contribute to result throughput the least. This data is written to disk once memory allotment for the query plan is exhausted, thus increasing the probability that new input data will help increase overall result throughput. The second method is a novel state manager module that is designed to fetch disk-resident data that will positively affect result throughput. Thus, the state manager directs each operator in the query pipeline to the most beneficial state during query runtime. These two methods assume that symmetric hash join, a common non-blocking join operator [17], [18], [21], is used to compute join results. This assumption makes the AdaptiveGlobalFlush method and the state manager module compatible with previous operators optimized for single-join query plans. Also, while these two methods are mainly designed to produce high early throughput in multi-join query plans, they also ensure the production of complete and exact query results, making them suitable for applications that do not tolerate approximations.

To the authors' knowledge, the state spilling approach [17] is the only work to have considered multijoin query plans. However, the work presented in this paper distinguishes itself from state-spilling and all other previous work through two novel aspects. First, the AdaptiveGlobalFlush algorithm attempts to maximize overall early result throughput by building its decisions over time based on a set of simple collected statistics that take into account both the data input and result output. Second, the state manager is a novel module that does not exist in previous work, and is designed specifically for multi-join query plans. The state manager has the ability to switch any join operator back and forth between joining in-memory data and disk-resident data during runtime based on the operation most beneficial for the set of pipelined join operators to produce early result throughput. When making its decision, the state manager module maintains a set of accurate, lightweight statistics that help in predicting the contribution of each join operator state. In general the contributions of this paper can be summarized as follows:

1) We propose a novel flushing scheme, AdaptiveGlobalFlush, applicable to any hash-based join algorithm in a multi-join query plan. AdaptiveGlobalFlush helps produce high early result throughput in multi-join query plans while being adaptive to the data arrival patterns.

2) We propose a novel state manager module that directs each join operator in the query pipeline to join either in-memory data or disk-resident data in order to produce high early result throughput.

3) We provide experimental evidence that our methods outperform the state-of-the-art join algorithms optimized for early results in terms of efficiency and throughput.

The rest of this paper is organized as follows: Section 2 highlights related work. Section 3 presents an overview of the methods proposed in this paper. The AdaptiveGlobalFlush method is described in Section 4. Section 5 presents the state manager module. Correctness of AdaptiveGlobalFlush and the state manager is covered in Section 6. Experimental evidence that our methods outperform other optimization techniques is presented in Section 7. Section 8 concludes the paper.

\section{ReLATED WORK}

The symmetric hash join [21] is the most widely used non-blocking join algorithm for producing early join results. However, it was designed for cases where all input data fits in memory. With the massive explosion of data sizes, several research attempts have aimed to extend the symmetric hash join to support disk-resident data. Such algorithms can be classified into three categories: (1) hash-based algorithms [10], [18], [19], [20] that flush in-memory hash buckets to disk either individually or in groups, (2) sort-based algorithms [15], [22] in which in-memory data is sorted before being flushed to disk, and (3) nestedloop-based algorithms [16] in which a variant of the traditional nested-loop algorithm is employed. Also, several methods have been proposed to extend these algorithms for other operators, e.g., MJoin [8] extends XJoin [20] for multi-way join operators while hashbased joins have been extended for spatial join operators [12]. However, these join algorithms employ optimization techniques that focus only on the case of a single join operator, with no applicable extension for multi-join query plans.

In terms of memory flushing algorithms, previous techniques can be classified to two categories: (1) Flushing a single hash bucket. Examples of this category include XJoin [20] that aims to flush the largest memory hash bucket regardless of its input source and RPJ [19] that evicts a hash bucket based on an extensive probabilistic analysis of input rates. (2) Flushing a pair of corresponding buckets from both data sources. Examples of this category include hash-merge join [18] that keeps the memory balanced between the input sources and state spilling [17] that attempts to maximize the overall early result 


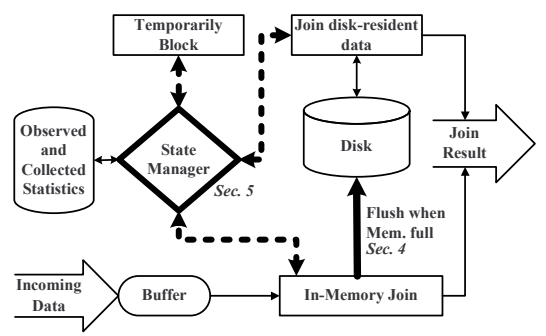

(a) Join Operation Overview

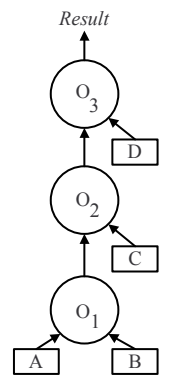

(b) Query Plan
Fig. 1. Join Overview and Example Query Plan

throughput of the query plan. Our proposed AdaptiveGlobalFlush method belongs to the latter category, as flushing a pair of buckets eliminates the need for extensive timestamp collection. Timestamps are necessary when flushing a single bucket to avoid duplicate join results [19], [20].

A family of previous work can be applied to streaming environments where input data is potentially infinite (e.g., [23], [24], [25]). Such algorithms aim to produce an approximate join result based on either load shedding [26], [27] or the definition of a sliding window over incoming streams [28]. Since our methods are concerned with finite input and exact query results, discussion of infinite data streams is outside the scope of this paper.

To the authors' knowledge the closest work to ours is PermJoin [29] and the state-spilling method [17]. PermJoin does not discuss specific methods for producing early results in multi-join query plans, only a generic framework. The basic idea behind state spilling is to score each hash partition group (i.e., symmetric hash buckets) in the query plan based on its current contribution to the query result. When memory is full, the partition group with the lowest score is flushed to disk. Once all inputs have finished transmission, the state-spill approach joins diskresident data using a traditional sort-merge join. Our proposed AdaptiveGlobalFlush method differs from the state-spilling flush approach in that it is predictive, taking into account both input and output characteristics to make an optimal flush decision. Furthermore, our proposed state manager is not found previous work, as it continuously operates during query runtime to place operators in an optimal state with regard to overall throughput. State-spill and other previous work consider changing operator state only when sources block or data transmission has terminated.

\section{Overview}

This section gives an overview of the two novel methods we propose for producing a high early result throughput in multi-join query plans. These methods are: (1) A new memory flushing algorithm, designed with the goal of evicting data from memory that will contribute to the result throughput the least, and optimized for overall (rather than local) early result throughput. (2) A state manager module designed with the goal of placing each operator in a state that will positively affect result throughput. Each operator can function in an in-memory, on-disk, or blocking state.

An overview of how our memory flushing algorithm and state manager can be added to existing non-blocking hash-based join algorithms is given in the state diagram in Figure 1(a). As depicted in the diagram, whenever a new tuple $R_{S}$ is received by the input buffer from source $S$ of operator $O$, the state manager determines how the tuple is processed. If $O$ is currently not in memory, the tuple $R_{S}$ will be temporarily stored in the buffer until $O$ is brought back to memory. Otherwise, $R_{S}$ will be immediately used to produce early results by joining it with in-memory data. Initially, all join operators function in memory. Once memory becomes full, the memory flushing algorithm frees memory space by flushing a portion of in-memory data to disk. During query runtime, each join operator may switch between processing results using either memory-resident or disk-resident data.

Memory Flushing. Most hash-based join algorithms optimized for early results employ a flushing policy to write data to disk once memory is full. In a multi-join query plan, policies that optimize for local throughput will likely perform poorly compared to policies that consider all operators together to optimize for overall throughput. For example, given the plan in Figure 1(b), if a local policy constantly flushes data from $O_{1}$, then downstream operators $\left(O_{2}\right.$ and $\mathrm{O}_{3}$ ) will be starved of data, degrading overall result throughput. We introduce a flushing policy, AdaptiveGlobalFlush, that can be added to existing hashbased join algorithms when part of a multi-join query plan. AdaptiveGlobalFlush opts to flush pairs of hash buckets, where flushing a bucket from an input source implies flushing the corresponding hash bucket from the opposite input at the same time. AdaptiveGlobalFlush evicts pairs of hash buckets that have the lowest contribution to the overall query output, using an accurately collected set of statistics that reflect both the data input and query output patterns. Details of AdaptiveGlobalFlush are covered in Section 4.

State Manager. The main responsibility of the state manager is to place each join operator in the most beneficial state in terms of producing high early result throughput. These states, as depicted in Figure 1(a) by rectangles, are: (1) Joining in-memory data, (2) Joining disk-resident data, or (3) Temporary blocking, i.e., not performing a join operation. As a motivating example, consider the query pipeline given in Figure 1(b). During query runtime, sources $A$ and $B$ may be transmitting data, while sources $C$ and $D$ are blocked. In this case, query results can only be generated from the base operator $O_{1}$. The overall query results produced by $O_{1}$ rely on the selectivity of the two operators above in the pipeline (i.e. $\mathrm{O}_{2}$ 


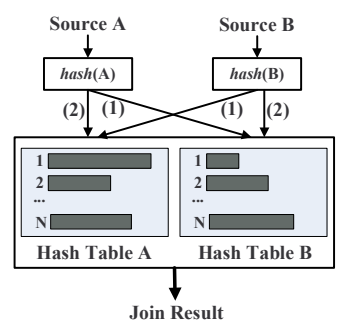

(a) Symmetric Hash Join

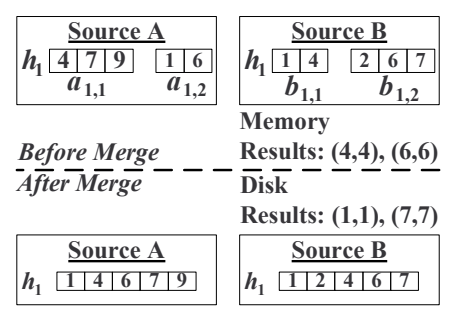

(b) On-Disk Merge
Fig. 2. Join and Disk Merge Examples

and $O_{3}$ ). If the selectivity of these operators is low, merging disk-resident data at either $\mathrm{O}_{2}$ or $\mathrm{O}_{3}$ may be more beneficial in maximizing the overall result throughput than performing an in-memory join at the base operator $O_{1}$. Thus, the state manager may decide to place $\mathrm{O}_{3}$ in the in-memory (i.e., default) state, $\mathrm{O}_{2}$ in the on-disk merge state, while $O_{1}$ is placed in a lowpriority state. Section 5 covers state manager details.

Architecture assumptions. We assume the in-memory join employs the symmetric hash join algorithm to produce join results [21]. Figure 2(a) gives the main idea of the symmetric hash join. Each input source $(A$ and $B$ ) maintains a hash table with hash function $h$ and $n$ buckets. Once a tuple $r$ arrives from input $A$, its hash value $h(r)$ is used to probe the hash table of $B$ and produce join results. Then, $r$ is stored in the hash bucket $h\left(r_{A}\right)$ of source $A$. A similar scenario occurs when a tuple arrives at source $B$. Symmetric hash join is typical in many join algorithms optimized for early results [18], [20], [21]. We also assume a join operator in the disk merge state employs a diskbased sort-merge join to produce results (e.g., see [17], [18]). Figure 2(b) gives an example of the sort-merge join in which partition group $h_{1}$ has been flushed twice. The first flush resulted in writing partitions $a_{1,1}$ and $b_{1,1}$ to disk while the second flush resulted in writing $a_{1,2}$ and $b_{1,2}$. Results from joining diskresident data are produced by joining (and merging) $a_{1,1}$ with $b_{1,2}$ and $a_{1,2}$ with $b_{1,1}$. Partitions $\left(a_{1,1}, b_{1,1}\right)$ and $\left(a_{1,2}, b_{1,2}\right)$ do not need to be merged, as they were already joined while residing in memory; the results produced from in-memory and on-disk operations are labeled in Figure 2(b). Flushing partition groups and using a disk-based sort-merge has the major advantage of not requiring timestamps for removal of duplicate results [17], [18]; once a partition group is flushed to disk it is not used again for in-memory joins.

In this work, we consider left-deep query plans with $m$ binary join operators $(m>1)$ where the base operator joins two streaming input sources while all other operators join one streaming input with the output of the operator below. An example query plan is given in Figure 1(b). We choose to study left-deep plans as they are common in databases. Our methods can apply to bushy trees, where our flush algorithm and state manager can be applied to leaf nodes of the plan. Future work will involve adapting our methods to non-leaf nodes in bushy multi-join query plans. Further, an extension of our framework to the case of query plans consisting of more than one m-way join operator is straightforward.

\section{Memory Flushing}

In online environments, most join operators optimized for early throughput produce results using symmetric hash join [18], [20], [21]. If memory allotment for the query plan is exhausted, data is flushed to disk to make room for new input, thus continuing the production of early results. In this section, we present the AdaptiveGlobalFlush algorithm that aims to produce a high early overall throughput for multijoin query plans. As discussed in Section 3, the AdaptiveGlobalFlush policy flushes partition groups simultaneously (i.e., corresponding hash partitions from both hash tables). The main idea behind AdaptiveGlobalFlush is to consider partition groups across all join operators in concert, by iterating through all possible groups, scoring them based on their expected contribution to the overall result throughput, and finally flushing the partition group with the lowest score, i.e., the partition group that is expected to contribute to the overall result throughput the least.

In general, a key success to any flushing policy lies in its ability to produce a high result throughput. In other words, the ability to flush hash table data that contributes the least to the result throughput. To avoid the drawbacks of previous flushing techniques [17], [18], [19], we identify the following three important characteristics that should be taken into consideration by any underlying flushing policy in order to $a c c u-$ rately determine in-memory data to flush:

- Global contribution of each partition group. Global contribution refers to the number of overall results that have been produced by each partition group. In multi-join query plans, the global contribution of each partition is continuously changing. For instance, any non-root join in the plan is affected by the selectivity of join operators residing above in the operator chain. These selectivities may change over time due to new data arriving and possibly being evicted due to flushing. The ability to successfully predict the global contribution of each partition group is a key to the success of a flushing policy in producing result throughput.

- Data arrival patterns at each join operation. Since data is transmitted through unreliable network connections, input patterns (i.e., arrival rates or delays) can significantly change the overall throughput during the query runtime. For example, higher data arrival rates imply that a join operator (and its partition groups) will contribute more to the overall throughput. Likewise, a lower data arrival rate implies less contribution to the overall throughput. Thus, a flushing policy 


\begin{tabular}{|l|l|l|}
\hline Class & Statistic & Definition \\
\hline \hline \multirow{3}{*}{ Size } & prtSize $_{S}$ & Size of hash partition $j$ for input $S$ \\
\cline { 2 - 3 } & grpSize $_{j}$ & Size of partition group $j$ \\
\cline { 2 - 3 } & tupSize $_{S}$ & Tuple size for input $S$ \\
\hline Input & input $_{t o t}$ & Total input count \\
\cline { 2 - 3 } & unique $_{j}$ & No. unique values in part. group $j$ \\
\cline { 2 - 3 } & prtInput $_{S j}$ & Input count at partition $j$ of input $S$ \\
\hline Output & obsLoc $_{j}$ & Local output of partition group $j$ \\
\cline { 2 - 3 } & obsGlo $_{j}$ & Global output of partition group $j$ \\
\hline
\end{tabular}

TABLE 1

Statistics

should consider the fluctuations of input patterns in order to accurately predict the overall throughput contribution of a partition group.

- Data Properties. Considering data properties is important as they directly affect the population, and hence the result production, for each partition group in a join operator. Such properties could be join attribute distribution or whether the data is sorted. For instance, if input data is sorted in a many-to-many or one-to-many join, a partition group $j$ may contribute a large amount to the overall result throughput within a time period $T$ as data in a sorted group (i.e., all tuples have join attribute $N$ ) may all hash to $j$. However, after time $T, j$ may not contribute to the result throughput for a while, as tuples from a new sorted group (i.e., with join attribute $N+1$ ) now hash to different partition $K$. Therefore, the flushing policy should be able to adapt during query runtime to data properties in order to accurately predict the population of a partition group.

\subsection{Adaptive Global Flush Algorithm}

AdaptiveGlobalFlush is a novel algorithm that aims to flush in-memory hash partition groups that contribute the least to the overall result throughput. The decisions made by the AdaptiveGlobalFlush algorithm mainly depend on the three characteristics just discussed; namely, global contribution, data arrival patterns, and data properties. The main idea of the AdaptiveGlobalFlush algorithm is to collect and observe statistics during a query runtime to help the algorithm choose the least useful partition groups to flush to disk. Flushing the least useful groups increases the probability that new input data will continue to help produce results at a high rate. We begin by discussing the statistical model that forms the "brains" behind the decision made by AdaptiveGlobalFlush to evict data from memory to disk. We then discuss the steps taken by AdaptiveGlobalFlush, and how it uses its statistical model, when flushing must occur. Throughout this section, we refer to the running example given in Figure 3 depicting a pipeline query plan with two join operators, Join $A B$ and Join $A B C$.

\subsubsection{Statistics}

The AdaptiveGlobalFlush algorithm is centered around a group of statistics observed and collected during the query runtime (summarized in Table 1). These statistics help the algorithm achieve its ultimate goal of determining, and flushing, the partition groups with the least potential of contributing to the overall result throughput. The collected statistics serve three distinct purposes, grouped into the following classes: Size statistics. The size statistics summarize the data residing inside a particular operator at any particular time. For each operator $O$, we keep track of the size of each hash partition $j$ at each input $S$, denoted prtSize ${ }_{S j}$. In Figure 3, the operator Join $A B$ has four partitions (i.e., two partition groups) with sizes prtSize $A 1=150$, prtSize $_{B 1}=30$, prtSize $_{A 2}=100$, prtSize $_{B 2}=15$. We also track the size of each pair of partitions grpSize $_{j}$, this value is simply the sum of the symmetric partitions grpSize $_{j}=$ prtSize $_{A j}+$ prtSize $_{B j}$. For example, in Figure 3, the size of partition groups at Join $A B$ are grpSize $1=150+30=180$ and grpSize $_{2}=100+15=115$. Finally, we keep track of the tuple size for the input data at each source $S$, assuming the tuple size is constant at each join input. In Figure 3, we observe a tuple size of tupSize $_{A}=10$ and tupSize $_{B}=8$.

Input statistics. The input statistics summarize the properties of incoming data at each operator. Due to the dynamic nature of data in an online environment, the input statistics are collected over a time interval $\left[t_{i}, t_{j}\right](i<j)$ and updated directly after the interval expiration (we cover these maintenance in Section 4.2). Specifically, for each operator $O$ we track the total number of input tuples received from all inputs, input $t_{t o t}$. Also, for each partition group $j$ in operator $O$, we track the statistic unique ${ }_{j}$ that indicates the number of unique join attribute values observed in $j$ throughout the query runtime. Finally, for each input $S$ and hash partition $j$, we track the number of tuples received at each partition $j$, prtInput ${ }_{S j}$. For the example in Figure 3, we assume the following values have been collected over a certain period of time: input $_{t o t}=100$, unique $_{2}=5$, prtInput $_{A 2}=6$, and prtInput $_{B 2}=4$.

Output statistics. Opposite of the input statistics, the output statistics summarize the results produced by the join operator(s). Like the input statistics, the output statistics are collected and updated over a predefined time interval. Specifically, for each partition group $j$ in each join operator, we track two output statistics, namely, the local output (obsLoc ${ }_{j}$ ) and the global output $\left(o b s G l_{j}\right)$. In a pipeline query plan, the local output of a join operator $O$ is the number of tuples sent from $O$ to the join above it in the operator tree. In Figure 3, all tuples that flow from $\operatorname{Join} A B$ to Join $A B C$ are considered local output of Join $A B$. The 


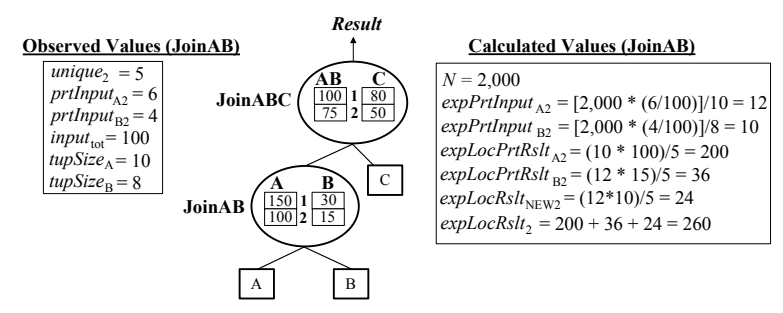

Fig. 3. Flush Example Plan

collected statistic $o b s L o c_{j}$ in operator $O$ is the number of tuples in the local output of $O$ produced by partition group $j$. The collected statistic obsGlo ${ }_{j}$ is the number of tuples in the global output (i.e., the local output of the root join operator, Join $A B C$ in Figure 3), produced by partition group $j$. To track this information, an identifier for the partition group(s) is stored on each tuple. Thus, each tuple stores a compact list of identifiers of the partition groups where it "lived" during query runtime. We note that storing identifiers versus recomputing the join to find the original hash group (as done in [17]) is a trade-off between storage overhead and computation costs. We choose the former method, which is more suitable for larger tuples, whereas a hash group re-computation algorithm can be implemented for smaller tuples. Once a tuple is output from the root join operator, it is considered to contribute to the global output, and hence the observed global output values for all the partition group identifier(s) stored on that tuple are incremented by one.

\subsubsection{AdaptiveGlobalFlush Algorithm}

The AdaptiveGlobalFlush algorithm takes as input a single parameter $N$, representing the amount of memory that must be freed (i.e., flushed to disk). The idea behind AdaptiveGlobalFlush is to evaluate each hash partition group at each join operator and score each group based on its expected contribution to the global output. The algorithm then flushes the partition group(s) with the lowest score until at least the amount of memory $N$ is freed and its data written to disk. We classify this process into four steps. In the rest of this section, we provide the ideas and intuition behind each step, and finish by providing a detailed look at the AdaptiveGlobalFlush algorithm.

Step 1: Input estimation. The input estimation step uses the input statistics (covered in Section 4.1.1) in order to estimate the number of tuples that will arrive at each operator input hash partition group $S_{j}$ until the next memory flush. Since each flushing instance aims to flush enough tuples to free an amount $N$ of memory, we know the next flush instance will occur after tuples with a combined size of $N$ enter the entire query plan. Thus, the goal of input estimation is to predict where (i.e., which partition group) each of these new tuples will arrive. We note that the size $N$ encompasses both new tuples streaming into the query plan, and intermediate results generated by joins lower in the operator tree. Estimating the number of tuples that will arrive at each partition group is the first step in gaining an idea of how "productive" a each group will be.

Step 2: Local output estimation. The local output estimation step uses the size statistics and input estimation from Step 1 in order estimate the contribution of each partition group to the local output of its join operator until the next flushing instance. The intuition behind this step is as follows. By tracking size statistics, we know, for each binary join operator, the size of each partition $j$ for each input $A$ and $B$. Further, through input estimation, we have an idea of how many new tuples will arrive at partition $A_{j}$ and $A_{b}$. Thus, to calculate local output, this step estimates a partitions contribution to the local output as the combination of three calculations: (1) The output expected from the newly arrived tuples at partition $A_{j}$ joining with tuples already residing in partition $B_{j}$. (2) The output expected from the newly arrived tuples at partition $B_{j}$ joining with tuples already residing in partition $A_{j}$. (3) The output expected from the newly arrived tuples at partition $A_{j}$ joining with the newly arrived tuples at partition $B_{j}$.

Step 3: Partition group scoring. The partition group scoring step uses the observed output statistics and the local output estimation from step 2 in order to score each partition group $j$ across all operators $O$, where the lowerst score implies that $j$ is the least likely to contribute to the overall global result output. The rationale for this score is to maximize the global output per number of tuples in the partition group. For example, consider two partition groups, $P$ with size 20 and $Q$ with size 10, each having an expected global output of 25. In this case, $Q$ would be assigned a better score as it has a higher global output count per number of tuples. The intuition behind this approach is that using local output estimation, we know how many local results will be produced by each partition group $j$. Through our observed output statistics, we can form a ratio $\frac{o b s L o c_{j}}{o b s G l o j}$ that allows us to estimate how many of these local results will become global results. We normalize the estimated global contribution by the partition size to derive a final score giving us the global output per partition size.

Step 4: Partition group flushing. The final partition group flushing step uses the scores from the partition group scoring in step 3 to flush partition groups to disk. This step simply chooses the lowest scoring partition group across all operators, and flushes it to disk. It iterates until tuples totaling the size $N$ have been flushed to disk.

Detailed Algorithm. We now turn to the details behind AdaptiveGlobalFlush. Algorithm 1 gives the pseudo-code for the algorithm, where each step previously outlined is denoted by a comment. The algorithm starts by iterating over all partition groups within all operators (Lines 2 to 4 in Algorithm 1).

Step 1 calculates an estimate for the number of new 


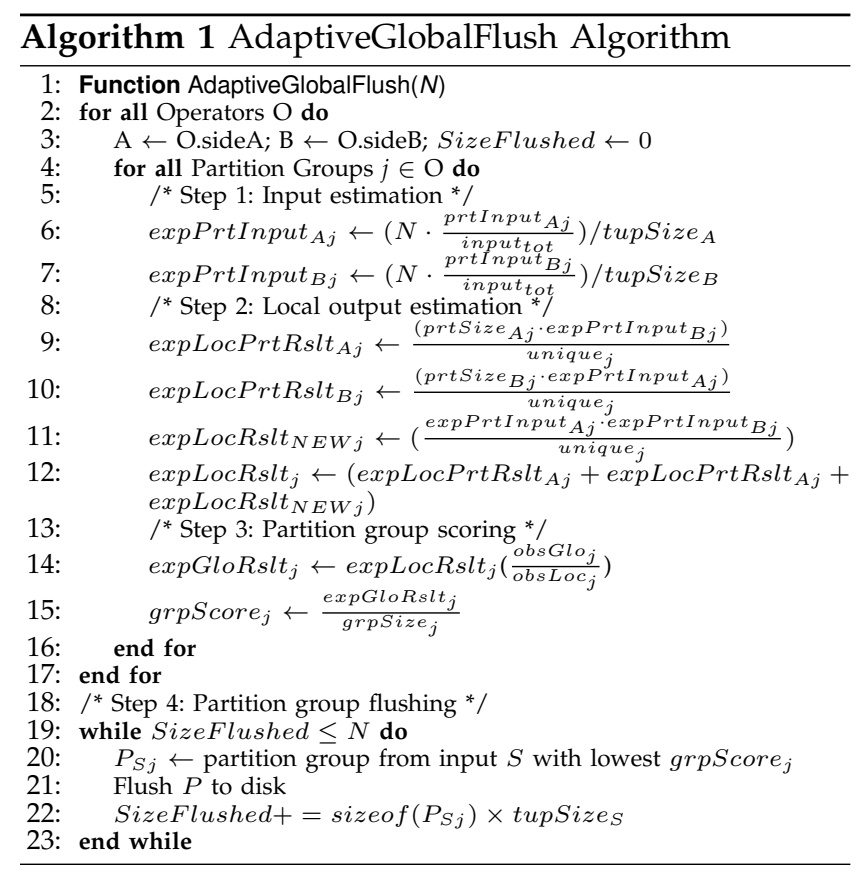

input tuples for each side $S$ of the partition group $j$, denoted as expPrtInput ${ }_{S j}$. This value is calculated by first multiplying the expected input size of all tuples in the query plan $N$ by the observed ratio that have hashed to side $S$ of $j$, formally prtInput $_{S j} /$ input $_{t o t}$. Second, this value is divided by the tuple size tupSize $_{S}$ for input $S$ to arrive at the number of tuples. As an example, in Figure 3, assuming that $N=2,000$ the expected input for each partition in group 2 of Join $A B$ is: $\operatorname{expPrtInput}_{A 2}=[2000 \times(6 / 100)] / 10=12$ and expPrtInput $_{B 2}=[2000 \times(4 / 100)] / 8=10$, assuming a tuple size of 10 and 8 at input $A$ and $B$, respectively.

Step 2 (Lines 9 to 12 in Algorithm 1) estimates a partition group's local output as follows. (1) The output from new tuples arriving at side $B$ hashing with existing data in partition side $A$ is computed by multiplying the two values expPrtInput ${ }_{B j}$ (from step 1) and prtSize ${ }_{A j}$ (a size statistic). However, to accommodate for the fact that a hash bucket $j$ may contain up to unique $_{j}$ different values, we divide the computed value by unique ${ }_{j}$. As an example, in Figure 3, this value for partition $A 2$ at $\operatorname{Join} A B$ is $\operatorname{expLocPrtRslt}_{A 2}=(10 \times 100) / 5=200$. (2) The output from new tuples arriving at side $A$ hashing with existing data in partition side $B$ is computed in a symmetric manner by exchanging the roles of $A$ and $B$. For example, in Figure 3 , this value for partition $B 2$ at Join $A B$ is expLocPrtRslt $B 2=(12 \times$ $15) / 5=36$. (3) The output estimate from newly arrived tuples from both $A$ and $B$ is computed by multiplying expPrtInput $_{A j}$ by expPrtInput exj $_{j}$ and dividing by unique $_{j}$. For example, in Figure 3 , this value for partition group 2 at Join $A B$ is $(12 \times 10) / 5=$ 24 . Finally, the local output estimation for partition group $j$, expLocRslt $j$ is the sum of the previously three computed values. In the example given in Figure 3, this value for partition group 2 at $\operatorname{Join} A B$ is expLocRslt L $_{2}=200+36+24=260$

Step 3 (Lines 14 to 15 in Algorithm 1) proceeds by first estimating the global output of partition group $j$ (denoted expGloRslt ${ }_{j}$ ) by multiplying expLocRslt ${ }_{j}$ by the ratio $\frac{o b s L o c_{j}}{o b s l_{0}}$. For example, assume that in Figure 3, the global/local ratio for partition group 2 in $\operatorname{Join} A B$ is 0.5 (i.e. half of its local output contributes to the global output). Then the expected global output for this partition group is expGloRslt $2=260 / 2=130$. Finally, the algorithm assigns a score ( grpScore $_{j}$ ) to partition group $j$ by dividing the expected global output by the size of the partition group (Line 15 in Algorithm 1).

In Step 4 (Lines 19 to 23 in Algorithm 1), the algorithm flushes partition groups with lowest score $\left(\right.$ grpScore ${ }_{j}$ ) until the desired data size $N$ is reached.

\subsection{Scoring Accuracy}

The quality of the AdaptiveGlobalFlush algorithm depends on the accuracy of the collected statistics. The size statistics (i.e., grpSize ${ }_{j}$ and prtSize $_{S j}$ ) are exact as they reflect the current cardinality that changes only when new tuples are received or memory flushing takes place. The input statistic unique ${ }_{j}$ is exact as it reflects a running total of the unique join attributes that arrive to partition group $j$. Also, we assume the statistics tupSize $_{S}$ is static, i.e., that tuple size will not change during runtime. However, other input statistics (i.e., prtInput $_{S j}$ and input $_{\text {tot }}$ ) and output statistics (i.e., $o b s L o c_{j}$, and $o b s G l_{j}$ ) are observed between a time interval $\left[t_{i}, t_{j}\right](i<j)$. In this section, we present three methods, namely, Recent, Average, and EWMA for maintaining such input and output statistics. Figure 4 gives an example of each method for the statistic $o b s L o c_{j}$ over four observations made from $t_{0}$ to $t_{4}$.

Recent. This method stores the most recent observation. In Figure 4, the Recent method stores only the value 60 observed between time period $t_{3}$ to $t_{4}$. The main advantage of this method is ease of use as no extra calculations are necessary. This method is not well suited for slow and bursty behavior.

Average. In this method, the average of the last $N$ observations is stored, where $N$ is a predefined constant. In Figure 4, the Average method stores the average of the last $N=3$ observations from time period $t_{1}$ to $t_{4}$, which is 77 . The average method is straightforward as it considers the last $N$ time periods to model the average behavior of the environment. It does not adhere to the fact that the recent behavior should weigh more than an old behavior.

Exponential Weighted Moving Average (EWMA). Using the EWMA method, each past observation is aged as follows: If the statistic at time period $t_{i}$ has value stat $t_{i}$ while at the time period $\left[t_{i}, t_{i+1}\right]$, we observe a new value ObsVal, then the value of the collected statistic is updated to be: stat $t_{i+1}=\alpha *$ 


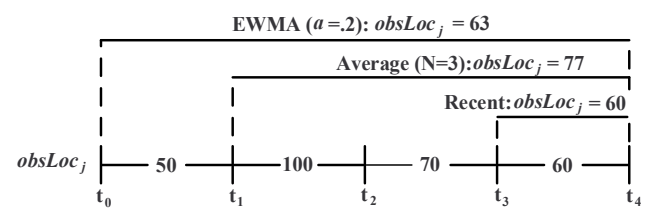

Fig. 4. Statistic Maintenance Sample

stat $_{i}+(1-\alpha) *$ ObsVal where $\alpha$ is a decay constant $(0<\alpha<1)$. By choosing a smaller $\alpha$, this method forgets past observations quickly and gives a greater weight to recent observations. The opposite is true for a larger $\alpha$. In Figure 4, the EWMA method (with $\alpha=0.2$ ) tracks all previous observations, but weights each new observation by a factor of 0.8 while weighting old observed values by 0.2. The EWMA method gives more accurate model for statistic maintenance.

\subsection{Discussion}

The statistics collected and calculated by AdaptiveGlobalFlush are designed to be lightweight due to its application in join processing. For instance, instead of calculating the exact intermediate input for all nonbase join operations coming from operator output lower in the query plan, we track the input independently at each input using prtInput $_{S j}$. By simplifying this statistical tracking, the flushing algorithm does not have to derive correlation between partition groups at each operator to calculate input to intermediate operators. As an example, consider a query plan with three joins as given in Figure 5(b). In order calculate the intermediate input to $J$ oin $A B C D$, we would need to derive the correlation between each partition group in $\operatorname{Join} A B$ to the partition groups in Join $A B C$ (i.e., where tuples in partition group $n$ of $\operatorname{Join} A B$ will hash in Join $A B C)$. Similarly, the correlation between groups in Join $A B C$ and Join $A B C D$ is needed. While calculating intermediate input this way would be more accurate than our approach, we feel the trade off between accuracy and algorithmic complexity is important in this case.

Comparison to State-of-the-Art Algorithms. Other flushing algorithms used by the state-of-the-art nonblocking join algorithms lack at least two of the three important aspects achieved by the PermJoin. For example, state-spilling flushing [17] only considers global contribution which is calculated based on the previous observed output, however, it does not adapt to data arrival patterns or data properties that affect the global throughput during the query runtime. The hashmerge join flushing algorithm [18] only considers data arrival patterns when attempting to keep hash tables balanced between inputs, however, global contribution and data properties are not taken into consideration. Finally, the RPJ flushing policy [19] adapts only to data arrival patterns and data properties by using a probability model to predict data arrival rates at each hash partition. However, global contribution is not considered by RPJ flushing. Furthermore, the RPJ policy

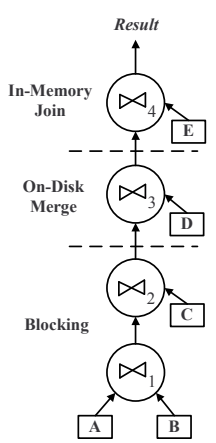

(a) State Example

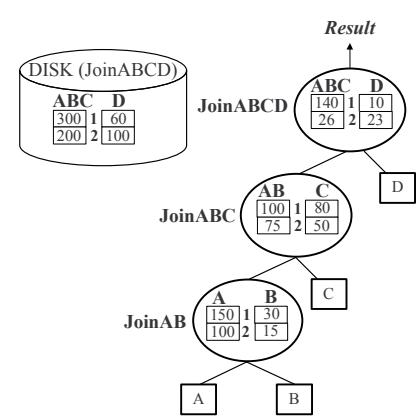

(b) Memory and Disk State

\section{Fig. 5. Multi-Join Plan Examples}

is designed only for single-join query plans with no straightforward extension for multi-join plans.

\section{The State Manager}

While the goal of the AdaptiveGlobalFlush algorithm is to evict data from memory that will contribute to the result throughput the least, the goal of the state manager is to fetch disk-resident data that will positively affect result throughput. The basic idea behind the state manager is, at any time during query execution, to place each operator in one of the following states: (1) In-memory join, (2) On-disk merge, or (3) Temporarily blocking (i.e., waiting for a join operator above in the pipeline to finish its merge phase). The state manager constantly monitors the overall throughput potential of the in-memory and ondisk states for each join operator. Overall throughput of intermediate operators (i.e., non-root operators) refers to the number of intermediate results produced that propagate up the pipeline to form an overall query result, whereas results at the root operator always contribute to the overall query result.

The state manager accomplishes its task by invoking a daemon process that traverses the query pipeline from top to bottom. During traversal, it determines the operator $O$ closest to the root that will produce a higher overall throughput in the on-disk state compared to its in-memory state. If this operator $O$ exists, it is immediately directed to its on-disk merge state to process results. Meanwhile, all operators below $O$ in the pipeline are directed to temporary block while all operators above $O$ in the pipeline continue processing tuples in memory. Figure 5(a) gives an example of a pipeline where the state manger finds that join operator $\triangle_{3}$ is the closest operator to the root capable of maximizing the query global throughput when joining disk-resident data rather than in-memory data. Thus, the state manger directs $\bowtie_{3}$ to the on-disk state while all the operators above $\bowtie_{3}$ (i.e., $\bowtie_{4}$ ) are directed to join in-memory data and all operators below $\bowtie_{3}$ (i.e., $\bowtie_{1}$ and $\bowtie_{2}$ ) are temporarily blocked.

The main rationale behind the state-manager approach is threefold. (1) Top-down traversal serves as an efficiency measure by shortcutting the search for 
operators where disk-resident operations are more beneficial to overall throughput than in-memory operations. (2) Each operator in a query pipeline produces an overall throughput greater than or equal to the lower operators in the query plan. Thus, increasing the overall throughput of an operator higher in the pipeline implies an increase in the overall throughput of the multi-join query. For example, in Figure 5(a) the state manager places $\bowtie_{3}$ in the on-disk merge state without traversing the pipeline further, as the overall result throughput contributions of $\bowtie_{1}$ and $\bowtie_{2}$ are bounded by $\pitchfork_{3}$, i.e., all intermediate results produced by $\bowtie_{1}$ and $\aleph_{2}$ must be processed by $\bowtie_{3}$ before propagating further. Thus, the overall result throughput produced by in-memory operations at $\bowtie_{1}$ and $\aleph_{2}$ cannot be greater than the overall in-memory throughput produced by $\aleph_{3}$. (3) While performing on-disk merge operations multiple operators in the query plan (e.g., $\bowtie_{3}$ and $\bowtie_{2}$ ) could also benefit result throughput, we feel placing a single operator in its on-disk state is sufficient due to the rationale just discussed. Furthermore, a single operator performing an on-disk join limits context switches, random I/O, and seeks that can result when multiple requests for disk operations are present.

In the rest of this section, we first provide the high-level state-manager algorithm. We then discuss the details behind the state manager's statistics and calculations. We finish with a discussion of the relative advantages of the state manager.

\subsection{State Manager Algorithm}

Algorithm 2 provides the pseudo code for the state manager module. As previously mentioned, the state manager algorithm performs a top-down traversal of all join operators in the pipeline. At each operator $O$, it compares the potential overall throughput for the in-memory and on-disk states. If the on-disk state is found to have a greater potential overall throughput at an operator $O$, the state manager directs $O$ to the on-disk merge state immediately. All operators above $O$ remain in the in-memory state, while all operators below $O$ are directed to temporarily block. In general, the state manager executes in two main stages (1) Evaluate states and (2) Direct operators. We now outline the functionality of each stage.

Stage 1: Evaluate states. The evaluate states stage performs a top-down traversal of the query plan, starting at the root operator (Line 2 in Algorithm 2). For the example given in Figure 5(b), the algorithm would traverse from JoinABCD down to JoinAB. The traversal ends when either an operator is found to switch to the on-disk state, or all operators have been visited (Line 4 in Algorithm 2). For the current operator $O$, the algorithm compares the overall throughput produced by the in-memory state to the throughput for the on-disk merge state (Lines 5 to 7 in Algorithm 2).

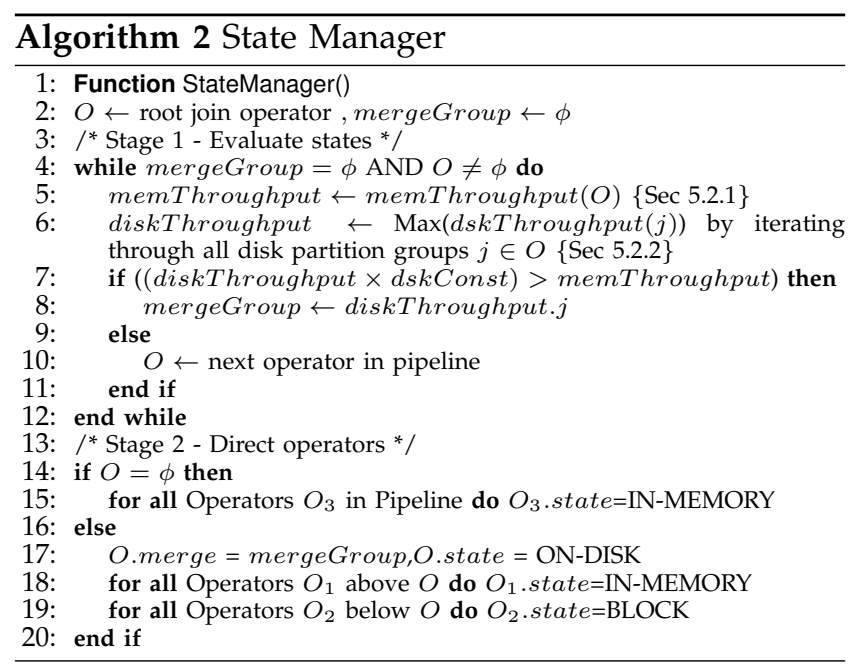

Details behind in-memory and on-disk throughput calculations are covered shortly in Section 5.2. This process begins by first calculating the in-memory throughput for $O$ (Line 5 in Algorithm 2). The ondisk merge state can only process one disk-resident partition group at a time. Thus, when comparing the on-disk throughput to in-memory throughput, the algorithm iterates over all disk-resident partition groups $j$ in $O$ to find the $j$ that will produce a maximal overall disk throughput (Line 6 in Algorithm 2). The algorithm then compares the overall throughput for an on-disk merge to the overall in-memory throughput for operator $O$. Since producing join results from disk is much slower than producing join results from memory, the state manager accounts for this fact by multiplying the calculated disk throughput by a disk throughput constant, $d s k C o n s t$. The value $d s k C o n s t$ is a tunable database parameter, and represents the maximum amount of tuples per time period (e.g., 1 second) that can be read from disk (Line 7 in Algorithm 2). If the on-disk throughput is maximal, the algorithm will mark the disk partition group (Line 8 in Algorithm 2). By marking $j$, the algorithm ends the traversal process and moves to the next stage. If the algorithm does not mark a partition group, traversal continues until all operators have been visited.

Stage 2: Direct operators This stage of the algorithm is responsible for directing each operator in the query pipeline to the appropriate state based on the analysis in stage 1 . Stage 2 starts by checking if the traversal in stage 1 found an operator $O$ to move to the on-disk state (Line 14 in Algorithm 2). If this is not the case, all operators in the query pipeline are directed to the inmemory state (Line 15 in Algorithm 2). Otherwise, we will direct $O$ to its on-disk state where it is asked to merge the marked disk partition group from stage 1 (Line 17 in Algorithm 2). Also, all operators above $O$ in the pipeline will be directed to the in-memory state while all operators below $O$ in the pipeline will be directed to block (Lines 18 to 19 in Algorithm 2). 


\subsection{Throughput Calculation}

From the high-level overview, we can see the core of the state manager is based on an accurate estimate of the throughput potential for the on-disk and in-memory states of each join operator in the query plan. We now cover the statistics and calculations necessary to estimate these throughput values.

\subsubsection{Memory Throughput}

The overall result throughput produced by the inmemory state at operator $O$ can be estimated as the sum of all observed global output values for its partition groups. Formally, this estimation is calculated as: memThroughput $(O)=\operatorname{memConst}\left(\sum_{j=1}^{|O|}\right.$ obsGlo $\left._{j}\right)$ where $|O|$ represents the number of partition groups in operator $O$ and $o b s G l o_{j}$ is the observed global output estimate over a predefined time interval (using a method from Section 4.2) for a partition group $j$ in $O$ (see Table 1). Much like our disk constant $d s k$ Const presented in Stage 1 of the state manager algorithm, the memory throughput calculation uses a tunable parameter memConst that models the maximum number of results that can be produced in-memory over a constant time period (e.g., 1 second).

\subsubsection{Disk Throughput}

The on-disk merge state allows only one disk-resident partition group to be merged at a time, meaning the overall disk throughput at an operator $O$ is represented by the disk group that produces the maximal overall throughput when merged. The overall disk throughput estimation is done per disk-resident group. Collected statistics. The disk throughput calculation relies on two exact statistics (i.e., they are not maintained using methods from Section 4.2). First, a size statistic that maintains, for each operator, the number of disk-resident tuples from input $S$ for each partition $j\left(d_{s k S i z e}{ }_{S j}\right)$. As with in the in-memory size statistics, $d_{s k S i z e}{ }_{S j}$ is an exact statistic as it represents the current cardinality. The other collected statistic maintains the current number of local results expected from an operator $O$ if it were to merge a disk partition group $j$ $\left(d s k L o c R s l t_{j}\right)$. The statistic $d s k L o c R s l t_{j}$ is initially set to zero, increases with every flush of partition group $j$, and reset to zero when the disk partition group $j$ is merged. Upon flushing partition group $j$ (with sides $A$ and $B$ ) to disk, the statistic $d s k L o c R s l t_{j}$ is increased by the number of results expected to be produced by $j$ which can be computed as follows: The flushed partition from side $B$ (partSize $_{B j}$; a collected in-memory size statistic), must eventually join with data already residing on disk (from a previous memory-flush) from side $A$ (dskSize Aj $_{j}$; a collected on-disk size statistic) while the flushed partition from $A$ (partSize $_{A j}$ ) joins with disk-resident data from $B\left(\right.$ dskSize $\left._{A j}\right)$. These estimations are possible because data is flushed in partition groups; new data being flushed from one partition side has not yet joined with disk-resident data from the other side. Since a partition group may contain tuples with multiple join attributes, the expected result must be divided by the unique number of join attributes observed in the partition group (unique $_{j}$; a collected in-memory input statistic). Formally, the statistic $d s k L o c R s l t_{j}$ is increased by the following value when a flush occurs: $\left(\left(d_{s k S i z e} e_{A j} \times\right.\right.$ partSize $\left._{B j}\right)+\left(\right.$ dskSize $_{B j} \times$ partSize $\left.\left._{A j}\right)\right) /$ unique $_{j}$. As an example, in Figure 5(b) (assuming unique $_{j}=5$ ), if partition group 1 from Join $A B C D$ were to be flushed to disk, the statistic dskLocRslt ${ }_{1}$ would be increased by: $((140 \times 60)+(300 \times 10)) / 5=2280$.

Overall disk throughput calculation Estimating the overall disk throughput for merging a disk partition group $j$ involves two steps. The first step calculates the expected global output for the merge $\left(d s k G l o R s l t_{j}\right)$. Similar to the computation made by the AdaptiveGlobalFlush algorithm, we can derive this value by multiplying the expected local output (dskLocRslt $j_{j}$; a collected on-disk statistic) by the observed global/local output ration (obsGlo ${ }_{j} / o b s L o c_{j}$; a collected in-memory statistic). Formally, this equation is: $d s k G l o R s l t_{j}=d s k L o c R s l t_{j} \times\left(o b s G l o_{j} / o b s L o c_{j}\right)$. In Figure 5(b), the global/local output ratio at Join $A B C D$ is 1 , as it is the root join operator. Therefore, if $d s k L_{o c R s l t}=2280$ for partition group 1 at JoinABCD, then sskGloRslt $_{1}$ is also 2280. The second step involves calculating a value for the number of global results expected per tuples read from disk. This calculation is similar to the scoring step of the AdaptiveGlobalFlush algorithm as we divide the expected global throughput by the size of the expected data read from disk. Formally, this equation for overall disk throughput is: $d_{s k T h r o u g h p u t}(j)=\left(\right.$ dskGloRslt $_{j} /\left(\right.$ dskSize $_{A j}+$ $\left.d s k S i z e_{B j}\right)$ ). For example, in Figure 5(b) (assuming dskLocRslt $1=2280$ ), the expected disk throughput for partition group 1 at $\operatorname{Join} A B C D$ is dskThroughput $(A B C D 1)=(2280 /(300+60))=6.33$. Also, since producing join results from disk is much slower than producing join results from memory, the estimation for the throughput of an on-disk merge must be bounded by the time needed to read data from disk. In Section 5.1, we described how the state manager accounts for this bound using the tunable parameter $d s k$ Const that represents disk throughput.

\subsection{Discussion}

One main goal of join algorithms optimized for early result throughput is the ability to produce results when sources block. When an operator is optimized for a single-join query plans, the decision to join data previously flushed to disk is straightforward as it is used to produce results only when both sources block [18], [19]. For multi-join queries, the statespilling algorithm [17] does not consider using disk- 
resident data to produce results while input is streaming to the query. Disk-resident data is used by statespilling in its cleanup phase, producing results when all input has terminated. Our proposed state manager goes beyond the idea of using disk-resident data only in the cleanup phase by invoking the disk merge operation when it is expected to increase the overall result throughput of the query. In this regard, the state manager is a novel algorithm for multi-operator query plans that uses disk-resident data to maintain a high result throughput outside the cleanup phase.

If the state manager finds that the on-disk state can produce a greater overall throughput than the inmemory state at an operator $O$, it immediately directs $O$ to enter its on-disk state. While this case is certainly true when both inputs to the operator $O$ block, it may be true otherwise. For instance, input rates at operator $O$ may slow down, decreasing its ability to produce overall results. If the operator $O$ is not a base operator, then one of these input rates is directly affected by the throughput of an operator located below in the pipeline. Thus, merging disk-resident data at operator $O$ is beneficial if it is guaranteed to generate tuples that will increase the overall result throughput.

Input Buffering. The state manager is able to place an operator in an on-disk or blocking state even if data is still streaming into the query plan at or below the on-disk operator. One consequence of this freedom is buffer overrun, i.e., exhausting the buffer memory for incoming tuples. One method to avoid buffer overrun is to ensure that switching operators to the on-disk and blocking phase will not cause buffer overrun. Using our collected statistics, we can easily perform this check. Let BufSpace be the available space in the buffer. Further, let DskTime be the time necessary to perform an on-disk merge. DskTime can be calculated as the time needed to read an on-disk partition group $j$. Using our disk size statistics, this value is $\frac{d_{s k S i z e} j+d s k \text { Size }_{B j}}{d s k \text { Const }}$, where dskConst is our tunable parameter modeling disk throughput. Also, recall from Section 4.1.1 that we measure (over time interval $\left.\left[t_{i}, t_{j}\right]\right)$ the incoming count to each partition $j$ for each join input $S$ as prtInput $_{S j}$. Let InputSum be the sum of all prtInput $_{S j}$ values for partitions residing in join operators at or below the operator about to be placed in an on-disk state. Then, we can model the time until the next buffer overrun as BufTime $=\frac{\left(t_{j}-t_{i}\right) \text { BufSpace }}{\text { InputSum }}$. Then, the state manager can reliably place the operators in the on-disk and blocking states without the threat of a buffer overrun if the value BufTime is greater than DskTime.

Cleanup. Because the AdaptiveGlobalFlush algorithm and the state manager are designed for multi-join query plans, results that are not produced during the inmemory or on-disk phase require special consideration. In this vein, we outline a cleanup process for each operator that is compatible with our framework to ensure that a complete result set is produced. In this phase, each operator begins cleanup by flushing all memory-resident data to disk after receiving an end-of-transmission (EOT) signal from both inputs. After this flush, all disk-resident partition groups are merged, producing a complete result set for that operator. The order of operator cleanup is important, due to the top-down dependency of pipeline operators. An example of this dependency is given in Figure 5(b). In order for Join $A B C$ to produce a complete result set, it must receive all input from source $C$ and $\operatorname{Join} A B$ before beginning the cleanup phase. Cleanup starts at the base operator and ends at the root of the query plan. To enforce cleanup order, we assume each operator in the chain can send an EOT signal to the next pipeline operator after completing its cleanup.

\section{Correctness}

Correctness of both AdaptiveGlobalFlush, and the state manager can be mapped to previous work. In terms of producing all results, we use symmetric hash join to produce in-memory results. Further we flush partition groups and use a variance of sort-merge join to produce disk results. Using these methods ensures that a single operator will produce all results [17], [18]. These methods also ensure that a single operator will produce a unique result exactly once in either the inmemory or on-disk states [18]. If the base operator in a query plan guarantees production of all results exactly once, the ordered cleanup phase ensures that all results from the base operator will propagate up the query plan. It follows that the this cleanup phase ensures that using our methods in a multi-join query plan will produce all results exactly once.

\section{EXPERIMENTAL RESULTS}

This section provides experimental evidence for the performance of the AdaptiveGlobalFlush algorithm and the state manager, by implementing them along with symmetric hash join [21]. We compare this implementation to optimizations used by the state-of-theart non-blocking join operators optimized for multijoin queries (i.e., state-spill [17]) as well as singlejoin queries (i.e., Hash-Merge Join (HMJ) [18] and RPJ [19]). Unless mentioned otherwise, all experiments use three join operations with four inputs $A$, $B, C$, and $D$ as given in Figure 5(b). All input arrival rates exhibit slow and bursty behavior. To model this behavior, input rates follow a Pareto distribution; widely used to model slow and bursty network behavior [30]. Each data source contains 300K tuples with join key attributes uniformly distributed in a range of $600 \mathrm{~K}$ values, where each join key "bucket" is assigned to a source with equal probability. Thus, given $M$ total join key buckets assigned to each join input $S$, there will be $X(X<M)$ overlapping join key buckets and $(M-X)$ non-overlapping join key 
buckets at each operator. Memory for the query plan is set to $10 \%$ of total input size.

For our proposed methods, statistical maintenance (see Section 4.1.1) is performed every five seconds $(t=5)$ using the EWMA method with an $\alpha$ value of 0.5. These parameters were determined with initial tuning experiments (not included for space reasons). For flushing policies, the percentage of data evicted is constant at $5 \%$ of total memory.

\subsection{Input Behavior}

Figure 6 gives the performance of AdaptiveGlobalFlush and the state manager (abbr. $\mathrm{AGF}+\mathrm{SM}$ ) compared to that of state-spill, HMJ, and RPJ when run in environments with four different input behaviors. In these tests, we focus on the first $100 \mathrm{~K}$ results produced, corresponding to early query feedback. Figure 6(a) gives the results for the default behavior (all inputs slow/bursty), and demonstrates the usefulness of AGF+SM. AdaptiveGlobalFlush is able to keep beneficial data in memory, thus allowing incoming data to produce a better overall early throughput. Meanwhile the state manager helps to produce results due to the slow and bursty input behavior where sources may block. The state-spill flush policy does not adapt to the slow and bursty input behavior in this case, and thus flushes beneficial data to disk early in the query runtime. Also, state-spill does not employ a state manager that is capable of finding on-disk data at each operator to help produce results. State-spill only invokes its disk phase once all inputs terminate. HMJ and RPJ, optimized for single-join queries, base their flushing decisions on maximizing output locally at each operator. However, these flushing policies are unaware of how data at each operator is being processed by subsequent operators in the pipeline, causing tuples to flood the pipeline that are unlikely to produce results higher in the query plan. HMJ invokes its disk phase once both inputs to an operator block. However, in a multi-join query, this scenario will likely occur only at the base operator, as one input for each intermediate operator is generated from a join operation residing below in the pipeline. In this case, disk results from the base operator can be pushed upward in the pipeline that will not produce results at subsequent operators, and cause flushing to occur higher in the pipeline when inputs are blocking, also degrading performance. On the other hand, RPJ performs better than HMJ due to its ability to switch from memory to disk operations based on its statistical model that predicts local throughput. For multi-join queries, a state manager is needed to manage processing at all operators in concert.

Figures 6(b) through $6(\mathrm{~d})$ give the performance of AGF+SM compared to state-spill HMJ, and RPJ for three, two, and one input(s) exhibiting slow and bursty behavior, respectively. In this left-right progres- sion, as the the inputs exhibit more of a steady behavior, the disk phase is used less, allowing us to pinpoint the relative behavior of each flushing policy only. While the performance of each algorithm converges from left to right, AGF+SM consistently outperform state-spill by at least 30\%, RPJ by $40 \%$, and HMJ by $45 \%$, in producing early results. AGF+SM also exhibit a constant steady behavior in both slow/bursty and steady environments. Finally, we note that these experiments show the benefit of optimization techniques for multi-join query plans, due to the performance of state-spill and AGF+SM compared to that of RPJ and HMJ. Also, due to the similar behavior of HMJ and RPJ compared to state-spill and AGF+SM, subsequent experiments use only HMJ to represent algorithms optimized for single-join query plans.

\subsection{Memory Size}

Figure 7 gives the time needed for $\mathrm{AGF}+\mathrm{SM}$, statespill, and HMJ to produce $100 \mathrm{~K}$ results for query plan memory sizes 5\% above and below the default memory size (i.e., 10\%) for two different environments. Figures $7(\mathrm{a})$ and $7(\mathrm{~b})$ give the performance for inputs exhibiting the default behavior, while figures 7(c) and 7(d) give the performance for inputs $A$ and B exhibiting steady behavior while $C$ and $D$ are slow and bursty. For smaller memory (Figures 7(a) and 7(c)), flushing occurs earlier causing more flushes during early result production. In this case, AdaptiveGlobalFlush is able to predict beneficial in-memory data and keep it in memory in order to maintain a high early overall throughput. Furthermore, when all inputs are slow/bursty (Figure 7(a)), the state manager helps in early result production. With larger memory (Figures $7(\mathrm{~b})$ and $7(\mathrm{~d})$ ), more room is available for incoming data, hence less flushing occurs early, meaning the symmetric-hash join is able to process most early results when memory is high. For both input behaviors, state-spill and AGF+SM perform relatively similar early on when more memory is available. Meanwhile, HMJ performs relatively better for a larger memory sizes, but its flushing and disk policy are still a drawback in multi-join query plans when inputs are slow/bursty compared to a steady environment.

\subsection{Data Flush Size}

Figure 8 give the performance for different data flush sizes. Figure 8(a) shows the time needed to produce the first $100 \mathrm{~K}$ results when smaller and larger percentages of total memory are evicted per flush operation, while Figure $8(\mathrm{~b})$ focuses on the specific case of $10 \%$ data flush. When the percentage is very low $(1 \%)$, flushes occur often, causing delays in processing. In general, writing less data to disk (in a reasonable range) during a flush operation leads to a greater 


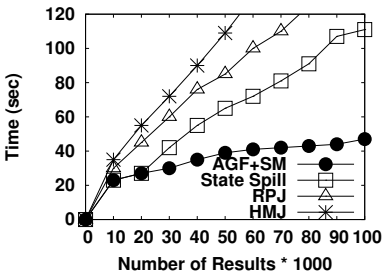

(a) $\mathrm{ABCD}_{B}$

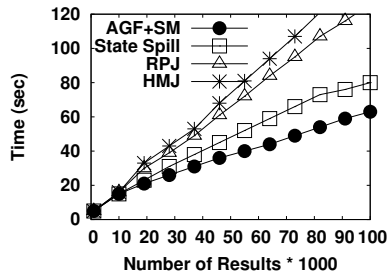

(b) $\mathrm{A}_{S}, \mathrm{BCD}_{B}$

Fig. 6. Input Behavior

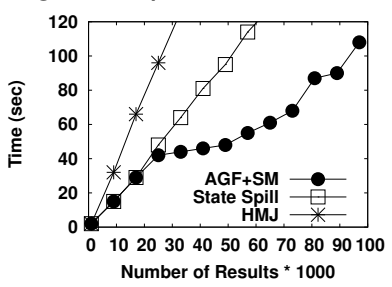

(a) $\mathrm{ABCD}_{B}$ Mem $5 \%$

Fig. 7. Query Memory Size

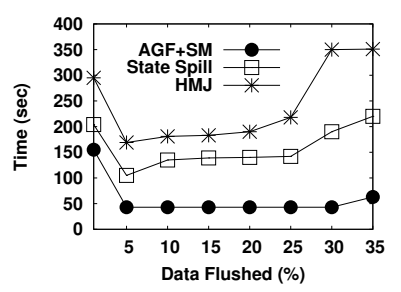

(a) Flush Progression

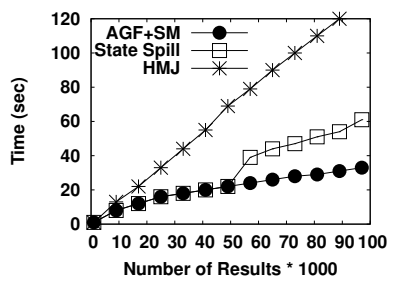

(b) $\mathrm{ABCD}_{B} \mathrm{Mem} 15 \%$

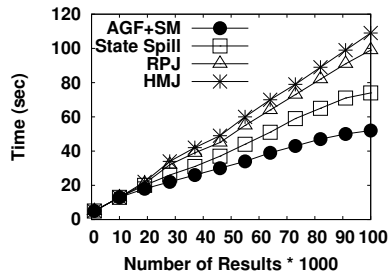

(c) $\mathrm{AB}_{S}, \mathrm{CD}_{B}$

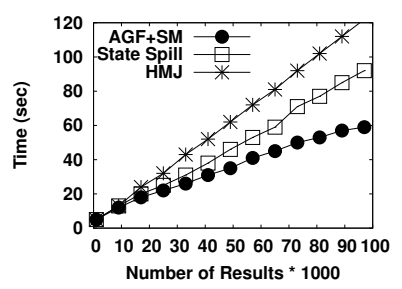

(c) $\mathrm{AB}_{S} \mathrm{CD}_{B} \mathrm{Mem} 5 \%$

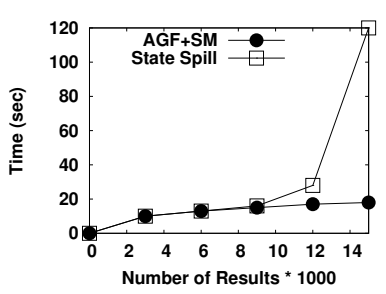

(a) 5-Join Query

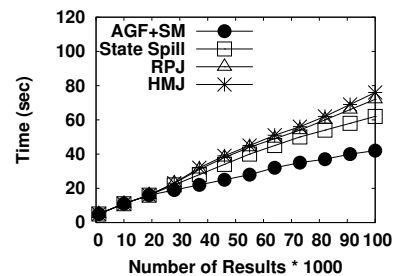

(d) $\mathrm{ABC}_{S}, \mathrm{D}_{B}$

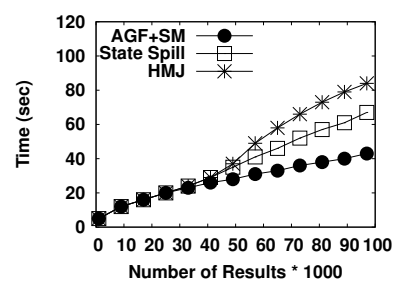

(d) $\mathrm{AB}_{S} \mathrm{CD}_{B}$ Mem $15 \%$

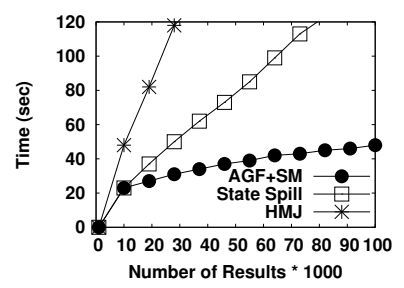

(b) Flush 10\%
Fig. 8. Data Flush Size

amount of early results as more data is available inmemory for arriving tuples. However, more overall flush operations are performed during query runtime leading to a large number of buckets on disk with low cardinality, which is less beneficial to the diskmerge phase. Writing more data to disk during a flush should lead to less amount of early results as less inmemory data is present for arriving tuples, while less flushes will be performed overall. Both state-spill and HMJ produce less early results in this case. However, AGF+SM performance remains stable. The reason for this performance is mainly due to the state manager. When AdaptiveGlobalFlush is required to write more tuples to disk at every flush, the state manager is able to quickly reclaim beneficial data to produce early results.

\subsection{Scalability}

Figure 9 gives the time needed for $\mathrm{AGM}+\mathrm{SM}$ and state-spill to produce the first $15 \mathrm{~K}$ results in 5 -join (6 inputs) and 6-join (7 inputs) query plans. In these tests, HMJ was omitted as no results were produced in the first 120 seconds. Due to the higher selectivity from more join operations, we plot the first $15 \mathrm{~K}$ results as cleanup begins earlier (steep slopes in figures) than in previous experiments. Here, $\mathrm{AGF}+\mathrm{SM}$ is able to delay the cleanup phase longer. If the AdaptiveGlobalFlush algorithm makes an incorrect decision and flushes beneficial data at an intermediate operator,

\section{Fig. 9. Number of Joins}

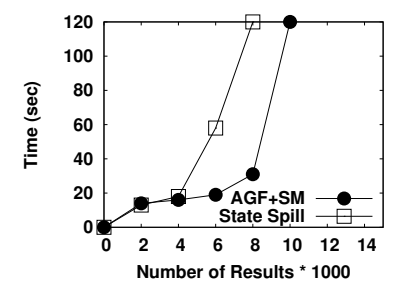

(b) 6-Join Query

the state manager is able to propagate these beneficial tuples by placing each operator in the in-memory or on-disk state in order to produce more results before cleanup. The state-spill algorithm does not make use of disk-resident data while producing these early results. Furthermore, state-spill relies only on its flushing algorithm that scores partition groups based on global vs. local contribution over size. This scoring method generally does not perform well with a large number of joins where selectivity is high in slow/bursty environments. Here, the ability to predict beneficial data in both the flush algorithm and disk states is important. Thus, state-spill exhibits lower relative performance in these cases.

\section{Conclusion}

This paper introduces a framework for producing high early result throughput in multi-join query plans. This framework can be added to existing join operators optimized for early results by implementing two novel methods. First, a new flushing algorithm, AdaptiveGlobalFlush, flushes data predicted to contribute to the overall early throughput the least by adapting to important input and output characteristics. This adaptation allows for considerable performance improvement over the methods used by other state-ofthe-art algorithms optimized for early results. Second, a novel module, termed a state manager, adaptively 
switches each operator in the multi-join query plan to process results in two states (in-memory or on-disk) to maximize the overall early throughput. The state manager is a novel concept, as it is the first module to consider the efficient use of disk-resident data in producing early results in multi-join queries. Extensive experimental results show that the proposed methods outperform the state-of-the-art join operators optimized for both single and multi-join query plans in terms of efficiency, resilience to changing input behavior, and scalability when producing early results.

\section{ACKNOWLEDGEMENTS}

This work is supported in part by the National Science Foundation under Grants IIS0811998, IIS0811935, IIS-0952977, CNS0708604, and by Microsoft Research Gifts

\section{References}

[1] G. Graefe, "Query Evaluation Techniques for Large Databases," ACM Computing Surveys, vol. 25, no. 2, pp. 73-170, 1993.

[2] P. Mishra and M. H. Eich, "Join Processing in Relational Databases," ACM Computing Surveys, vol. 24, no. 1, pp. 63113, 1992.

[3] L. D. Shapiro, "Join Processing in Database Systems with Large Main Memories," TODS, vol. 11, no. 3, pp. 239-264, 1986.

[4] T. Urhan, M. J. Franklin, and L. Amsaleg, "Cost Based Query Scrambling for Initial Delays," in SIGMOD, 1998.

[5] G. Abdulla, T. Critchlow, and W. Arrighi, "Simulation Data as Data Streams," SIGMOD Record, vol. 33, no. 1, pp. 89-94, 2004.

[6] J. Becla and D. L. Wang, "Lessons Learned from Managing a Petabyte," in CIDR, 2005.

[7] R. S. Barga, J. Goldstein, M. Ali, and M. Hong, "Consistent Streaming Through Time: A Vision for Event Stream Processing," in CIDR, 2007.

[8] S. Viglas, J. F. Naughton, and J. Burger, "Maximizing the Output Rate of Multi-Way Join Queries over Streaming Information Sources," in VLDB, 2003.

[9] D. T. Liu, M. J. Franklin, G. Abdulla, J. Garlick, and M. Miller, "Data-Preservation in Scientific Workflow Middleware," in SSDBM, 2006.

[10] Z. G. Ives, D. Florescu, M. Friedman, A. Y. Levy, and D. S. Weld, "An Adaptive Query Execution System for Data Integration," in SIGMOD, 1999.

[11] D. J. DeWitt and J. Gray, "Parallel Database Systems: The Future of High Performance Database Systems," Commununications of ACM, CACM, vol. 35, no. 6, pp. 85-98, 1992.

[12] G. Luo, J. F. Naughton, and C. Ellmann, "A Non-Blocking Parallel Spatial Join Algorithm," in ICDE, 2002.

[13] M. A. Hammad, W. G. Aref, and A. K. Elmagarmid, "Stream Window Join: Tracking Moving Objects in Sensor-Network Databases," in SSDBM, 2003.

[14] G. S. Iwerks, H. Samet, and K. P. Smith, "Maintenance of KNN and Spatial Join Queries on Continuously Moving Points," TODS, vol. 31, no. 2, pp. 485-536, 2006.

[15] J.-P. Dittrich, B. Seeger, D. S. Taylor, and P. Widmayer, "Progressive Merge Join: A Generic and Non-blocking Sort-based Join Algorithm," in $V L D B, 2002$.

[16] P. J. Haas and J. M. Hellerstein, "Ripple Joins for Online Aggregation," in SIGMOD, 1999.

[17] B. Liu, Y. Zhu, and E. A. Rundensteiner, "Run-time operator state spilling for memory intensive long-running queries," in SIGMOD, 2006.

[18] M. F. Mokbel, M. Lu, and W. G. Aref, "Hash-Merge Join: A Non-blocking Join Algorithm for Producing Fast and Early Join Results," in ICDE, 2004.
[19] Y. Tao, M. L. Yiu, D. Papadias, M. Hadjieleftheriou, and N. Mamoulis, "RPJ: Producing Fast Join Results on Streams through Rate-based Optimization," in SIGMOD, 2005.

[20] T. Urhan and M. J. Franklin, "XJoin: A Reactively-Scheduled Pipelined Join Operator," IEEE Data Engineering Bulletin, vol. 23, no. 2, pp. 27-33, 2000.

[21] A. N. Wilschut and P. M. G. Apers, "Dataflow Query Execution in a Parallel Main-Memory Environment," in PDIS, 1991.

[22] J.-P. Dittrich, B. Seeger, D. S. Taylor, and P. Widmayer, "On producing join results early," in PODS, 2003.

[23] J. Kang, J. F. Naughton, and S. Viglas, "Evaluating Window Joins over Unbounded Streams," in ICDE, 2003.

[24] U. Srivastava and J. Widom, "Memory-Limited Execution of Windowed Stream Joins," in $V L D B, 2004$.

[25] J. Xie, J. Yang, and Y. Chen, "On Joining and Caching Stochastic Streams," in SIGMOD, 2005

[26] N. Tatbul, U. Çetintemel, S. B. Zdonik, M. Cherniack, and M. Stonebraker, "Load Shedding in a Data Stream Manager," in $V L D B, 2003$.

[27] Y.-C. Tu, S. Liu, S. Prabhakar, and B. Yao, "Load Shedding in Stream Databases: A Control-Based Approach," in VLDB, 2006.

[28] M. A. Hammad, M. J. Franklin, W. G. Aref, and A. K. Elmagarmid, "Scheduling for Shared Window Joins over Data Streams," in $V L D B, 2003$.

[29] J. J. Levandoski, M. E. Khalefa, and M. F. Mokbel, "PermJoin: An Efficient Algorithm for Producing Early Results in Multijoin Query Plans," in ICDE, 2008.

[30] M. E. Crovella, M. S. Taqqu, and A. Bestavros., Heavy-tailed probability distributions in the world wideweb. Chapman Hall, 1998, ch. A practical guide to heavy tails: statistical techniquesand applications.

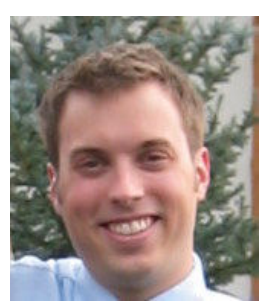

Justin J. Levandoski received his Bachelor's degree at Carleton College, MN, USA in 2003, and his Master's degree at the University of Minnesota, MN, USA in 2008. $\mathrm{He}$ is pursuing a $\mathrm{PhD}$ degree in computer science at the University of Minneosta. His $\mathrm{PhD}$ research focuses on embedding preference and contextual-awareness inside the database engine. His general research interests focus on extending database technologies to handle emerging applications.

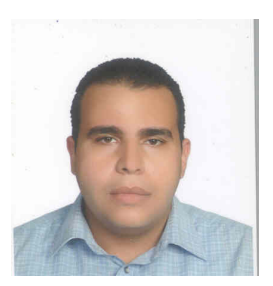

Mohamed E. Khalefa received the BS and MS degrees in Computer Science and Engineering in 2003 and 2006, respectively, from Alexandria University, Egypt. He is currently pursuing a $\mathrm{PhD}$ in database systems at the University of Minnesota. His research interests include non-traditional query processing, preference queries, uncertain data processing, and cloud computing.

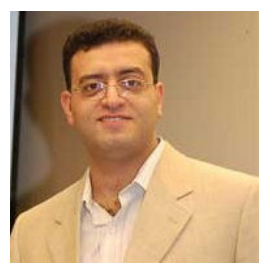

Mohamed F. Mokbel (Ph.D., Purdue University, 2005, MS, B.Sc., Alexandria University, 1999, 1996) is an assistant professor in the Department of Computer Science and Engineering, University of Minnesota. His main research interests focus on advancing the state of the art in the design and implementation of database engines to cope with the requirements of emerging applications (e.g., location-based applications and sensor networks). His research work has been recognized by two best paper awards at IEEE MASS 2008 and MDM 2009. Mohamed is a recipient of the NSF CAREER award 2010. Mohamed has actively participated in several program committees for major conferences including ICDE, SIGMOD, VLDB, SSTD, and ACM GIS. He is/was a program co-chair for ACM SIGSPATIAL GIS 2008, 2009, and 2010. Mohamed is an ACM and IEEE member and a founding member of ACM SIGSPATIAL. For more information, please visit http://www.cs.umn.edu/ mokbel. 